\title{
COMMENT
}

Check for updates

\section{The global rise of 3D printing during the COVID-19 pandemic}

\author{
Yu Ying Clarrisa Choong ${ }^{1 *}$, Hong Wei Tan $\mathbb{1}^{2 *}$, Deven C. Patel ${ }^{3}$, \\ Wan Ting Natalie Choong', Chun-Hsien Chen (1)', Hong Yee Low², Ming Jen Tan (1)', \\ Chandrakant D. Patel ${ }^{1,4}$ and Chee Kai Chua $\mathbb{1}^{2 凶}$
}

3D printing enables on-demand solutions for a wide spectrum of needs ranging from personal protection equipment to medical devices and isolation wards. This versatile technology is suited to address supply-demand imbalances caused by socio-economic trends and disruptions in supply chains.

The global uncertainty created by the COVID-19 pandemic has plunged the world into a crisis that is still unfolding. Logistical challenges owing to disruptions in manufacturing and transportation, together with pushbacks against globalization and free trade, have constrained supply chains, resulting in critical shortages of essential goods. Healthcare systems are on a war footing to increase their capacity of beds, supplies and trained workers. Crisis-response efforts are in motion to alleviate shortages of much-needed medical supplies.

There is a need for factories to manufacture, on demand, materials and devices for a range of essential services, in particular for healthcare. In this context, a resilient advanced manufacturing network enabled by a distribution of 3D-printing factories has great potential. Indeed, these 'art-to-part' factories can be co-located at hospitals and transportation hubs to quickly serve the needs of the medical profession. 3D printing ${ }^{1}$ has redeployed its capabilities in the crucible of COVID-19 responses, demonstrating its competitive advantage in this emergency situation.

\section{Harnessing the advantages of 3D printing}

The digital versatility and quick prototyping of 3D printing empowers a swift mobilization of the technology and hence a rapid response to emergencies. Even during severe disruptions in supply chains, critical parts can be manufactured on-demand by any decentralized 3D-printing facility in the world by leveraging designs shared online. Moreover, the additive nature of $3 \mathrm{D}$ printing enables product customization and complex designs. The broad spectrum of 3D-printing applications in the fight against COVID-19 includes personal protective equipment $(\mathrm{PPE})^{2-4}$, medical ${ }^{5,6}$ and testing devices ${ }^{7}$, personal accessories, visualization aids and emergency dwellings (FIG. 1).

Owing to severe shortages of ventilator machines, continuous positive airways pressure (CPAP) machines have been used as substitutes for COVID-19 patients who require sub-intensive therapy. An Italian engineering company, Isinnova, came up with a 3D-printable mask connector design, the Charlotte valve, which is specially designed to fit and connect Decathlon's Easybreath snorkelling masks to CPAP machines. 3D printing also serves as an efficient manufacturing option to keep up with the demand for nasopharyngeal swabs. The 3D-printed swabs are fabricated with complex tip structures for enhanced sample collection efficacy, hence eliminating the need to apply flocks at the tips.

Personalized 3D-printed face masks that provide an ergonomic fit are enabled by the mass customization capability of 3D printing combined with the use of 3D scanning. Moreover, in view of addressing the environmental concerns on medical waste accumulated from disposable PPE, 3D printing offers promising solutions to conserve precious resources by advocating recyclable materials and reusability of respirators and filters.

$3 \mathrm{D}$ printing is also being used to provide training and visualization aids for healthcare workers to cope with the limited pool of trained personnel. The ability to replicate complex human anatomy in highly accurate models enables the 3D printing of life-sized medical manikins to complement training materials and allow healthcare workers to practice COVID-19 swab testing procedures with standard medical-grade swabs. Some parts of the manikin are printed with transparent materials to reveal the internal structures of the nasal cavity, throat and mouth. Trainers can demonstrate the procedures clearly while emphasizing special precautions to minimize discomfort in patients.

Finally, 3D printing has been used to fabricate temporary emergency dwellings to isolate those under quarantine, relieving the overloaded medical infrastructures. Compared to traditional construction methods, 3D printing of buildings usually requires shorter building times and lower labour costs, and can use more environmentally friendly raw materials. 3D-printed dwellings can also be easily transported and deployed to areas where they are most needed. 


\section{Addressing the unaddressed concerns}

While the course of the pandemic remains unclear and the $3 \mathrm{D}$-printing community is working on developing even more printable devices, safeguarding lives and users' wellbeing remains a priority. There are growing concerns around the potential risks associated with the medical use of 3D-printed devices during this pandemic. To maintain a high level of confidence in this technology, it is important to ensure the technological suitability and accountability of new approved designs and certified materials to harness the benefits of $3 \mathrm{D}$ printing.

Federal agencies and government authorities have implemented regulations and drawn up guidelines ${ }^{8}$ that manufacturers of 3D-printed medical devices must comply with. An international risk classification system was introduced for 3D-printed medical devices, and essential principles of safety and performance now have to be met. All manufacturing and post-processing activities have to be documented and performed within an ISO 13485-compliant quality-management system, and final printed devices have to be evaluated for biocompatibility based on international standards (such as ISO10993-1). Building a strong framework for accountability and implementing guidelines supported by regulators and even

3D printing applications for COVID-19

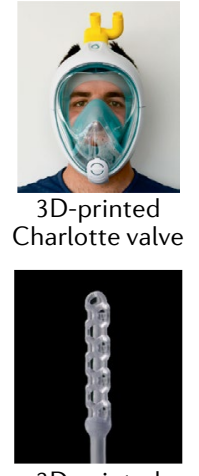

3D-printed NP swab

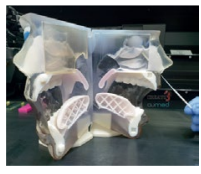

3D-printed medical manikin

\section{Medical devices \\ - Ventilator valves \\ - Mask connectors for \\ CPAP and BiPAP \\ - Emergency respiration \\ device \\ - Non-invasive PEEP mask}

\section{Testing devices}

- Nasopharyngeal (NP)

swabs

\section{Training and}

visualization aids

- Medical manikins

- Bio-models

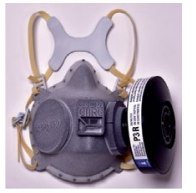

3D-printed respirator

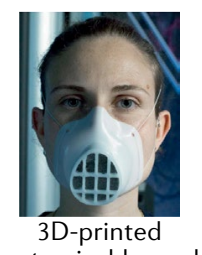

customizable mask

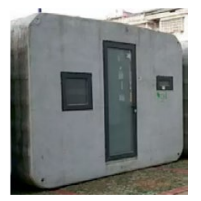

3D-printed

isolation wards
Personal protective equipment (PPE)

- Face shield

- Respirators

- Metal respirator filters

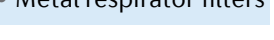

Personal accessories

- Face masks

- Mask fitters

- Mask adjusters

- Door openers

Emergency dwellings

- Isolation wards
Fig. 1 Applications of 3D printing in the fight against COVID-19. 3D printing is being used to provide many different solutions to the challenges posed by the COVID-19 pandemic, ranging from personal protective equipment (PPE) to emergency dwellings to isolate patients. CPAP: continuous positive airways pressure; BiPAP: Bilevel positive airway pressure. 3D-printed respirator image adapted from https://www.ciirc.cvut.cz/ covid-2/, copyright CIIRC CTU 2020; image of 3D-printed mask from https:// www.3dwasp.com/en/3d-printed-mask-from-3d-scanning/, courtesy of WASP\#3dwasp 3dwasp.com; image of 3D-printed isolation ward adapted with permission from http:// www.winsun3d.com/En/News/news_inner/id/543, Yingchuang Building Technique (Shanghai) Co., Ltd (WinSun); image of the 3D-printed medical manikin from https:// creatz3d.com.sg/3d-printed-medical-manikins-become-effective-training-aids-forrespiratory-swab-collection/, courtesy of Creatz3D \& AuMed https://www.creatz3d. com.sg; image of 3D-printed swab from https://envisiontec.com/wp-content/uploads/ 2020/04/EnvisionTEC-NP-Swabs.pdf, courtesy of EnvisionTEC; image of Charlotte valve adapted with permission from https://www.isinnova.it/easy-covid19/, Isinnova. governments can help to establish greater confidence in 3D-printed medical devices.

Regulations, however, tend to lag behind innovation, which can contribute to a culture that places undue reliance on procedures, checklists and rules rather than core principles and an ethical culture. For example, the convenience of digital file sharing could facilitate high levels of copyright infringement. An ethical approach is needed to avoid heightened tensions between different groups with competing interests and delayed emergency responses as a consequence of copyright infringement. Post-market duties and obligations, which include ensuring that the printed devices can function as intended and reporting previously unforeseen device defects or malfunctions, can build broader ethics and accountability frameworks around the technology.

$3 \mathrm{D}$ printer owners around the world, from hobbyists to industry users, have been amplifying their commitment with innovative solutions to help in the global COVID-19 supply efforts. With lives at stake, it is paramount that the $3 \mathrm{D}$-printing community works in parallel with medical professionals to avoid creating undue risks to public health. Collaborations between $3 \mathrm{D}$ printer users and medical experts can ensure the safe and effective performance of 3D-printed medical devices and translate the good intentions of individuals into meaningful contributions.

\section{D-printing landscape after COVID-19}

In the heat of the COVID-19 pandemic, 3D printing has stepped up to become a vital technology to support improved healthcare and our general response to the emergency. The crisis has highlighted how $3 \mathrm{D}$ printing can be at the base of a greener and more environmentally friendly future. $3 \mathrm{D}$ printing, thanks to the possibility to produce parts on demand, can reduce waste and inventory. Its inherent flexibility and the possibility to modify designs available online are unleashing creative and sustainable solutions that can carry the technology forward in the 'new normal.'

Supply chains are expected to be shorter and more fragmented as the global economy reopens. These changes will result in different manufacturing procedures with more partnerships in an open additive-manufacturing ecosystem. The main attributes of 3D printing - a high level of customization for specific needs and decentralized manufacturing - are likely to bring about local microgrids of 3D-printing factories. Digitization will continue to transform 3D-printing machines into key parts of the Internet of Things and Industry 4.0 environments in the post-pandemic, cyber-physical age.

1. Chua, C. K. \& Leong, K. F. 3D Printing and additive manufacturing principles and applications. 5th edn (World Scientific Publishing, 2017).

2. He, H., Gao, M., Illês, B. \& Molnar, K. 3D printed and electrospun, transparent, hierarchical polylactic acid mask nanoporous filter. Int. J. Bioprint. 6(4) https://dx.doi.org/10.18063/ijb.v6i4.278 (2020).

3. Bishop, E. G. \& Leigh, S. J. Using large-scale additive manufacturing (LSAM) as a bridge manufacturing process in response to shortages in PPE during the COVID-19 outbreak. Int. J. Bioprint. 6(4) https:// doi.org/10.18063/ijb.v6i4.281 (2020).

4. Celik, H. K. et al. Design and additive manufacturing of a medical face shield for healthcare workers battling coronavirus (COVID-19). Int. J. Bioprint. 6(4) https://doi.org/10.18063/ijb.v6i4.286 (2020).

5. lyengar, K., Bahl, S., Raju, V. \& Vaish, A. Challenges and solutions in meeting up the urgent requirement of ventilators for COVID-19 patients. Diabetes \& Metabolic Syndrome: Clinical Research \& Reviews 14, 499-501 (2020). 
6. Armani, A. M., Hurt, D. E., Hwang, D., McCarthy, M. C. $\&$ Scholtz, A. Low-tech solutions for the COVID-19 supply chain crisis. Nat. Rev. Mater. 5, 403-406 (2020).

7. Callahan, C. J. et al. Open development and clinical validation of multiple 3D-printed sample-collection swabs: rapid resolution of a critical COVID-19 testing bottleneck. medRxiv https://doi.org/10.1101/2020.04.14.20065094 (2020).

8. Bhat, S., Venkatesh, M., Balamuralidhara, V. \& Kumar, T. P. Comparison of 3D printing in USA, Europe and Australia and IPR. J. Pharm. Sci. \& Res. 11, 2515-2520 (2019).

\section{Acknowledgements}

This research is conducted in collaboration with HP Inc. and supported by National Research Foundation (NRF) Singapore and the Singapore Government through the Industry Alignment Fund, Industry Collaboration Projects Grant (I1801E0028). This work is also supported by the Chen Tsang Man chair professorship at Singapore University of Technology and Design (SUTD). The authors acknowledge HP-NTU Digital Manufacturing Corporate Lab and Digital Manufacturing and Design Centre (DManD). The authors thank AuMed, Creatz3D, CIIRC CTU, EnvisionTEC, Isinnova, WASP and Yingchuang Building Technique (Shanghai) Co., Ltd. (WinSun) for the graphical images.
Author contributions

Y.Y.C. Choong \& H.W. Tan: conceptualization, visualization, writing original draft, review $\&$ editing. D.C. Patel, W.T.N. Choong, C.-H. Chen, H.Y. Low \& M.J. Tan: project administration, review $\&$ editing. C.D. Patel \& C.K. Chua: supervision, review $\&$ editing.

\section{Competing interests}

The authors declare no competing interests.

\section{RELATED LINKS}

3D printed swabs: https://envisiontec.com/wp-content/uploads/2020/04/ EnvisionTEC-NP-Swabs.pdf

3D printing of life-sized medical manikins: https://creatz3d.com.sg/ 3d-printed-medical-manikins-become-effective-training-aids-forrespiratory-swab-collection/

An international risk classification system: https://www.fda.gov/medicaldevices/3d-printing-medical-devices/fdas-role-3d-printing

Charlotte valve: https://www.isinnova.it/easy-covid19/

Personalized 3D-printed face masks: https://www.3dwasp.com/en/ 3d-printed-mask-from-3d-scanning/

Respirators: https://www.ciirc.cvut.cz/covid-2/

Temporary emergency dwellings: http://www.winsun3d.com/En/News/ news_inner/id/543 